\title{
CAIS
}

\section{Padovan and Perrin Hybrid Number Identities}

\author{
Renata Passos Machado Vieira ${ }^{1 *}$, Milena Carolina dos Santos Mangueira ${ }^{2}$, Francisco Regis \\ Vieira Alves ${ }^{3}$, Paula Maria Machado Cruz Catarino ${ }^{4}$
}

\begin{abstract}
This work investigates the numbers of Padovan and Perrin hybrids. At first, the hybrid numbers, the sequences in the hybrid form and their matrix forms are ordered as studied sequences. Thus, it was possible to display the negative index hybrids, define some identities belonging to these hybrid sequences, develop novel theorems and present them as binomial sums of the Padovan and Perrin hybrids.

Keywords: Identities, hybrid numbers, Padovan sequence, Perrin sequence 2010 AMS: Primary 11B35, Secondary 11B39

${ }^{1}$ Department of Mathematics, Federal Institute of Education, Science and Techonology of State of Ceara - IFCE, ORCID:0000-0002-1966-7097

${ }^{2}$ Department of Mathematics, Federal Institute of Education, Science and Techonology of State of Ceara - IFCE, ORCID:0000-0002-4446-155X

${ }^{3}$ Department of Mathematics, Federal Institute of Education, Science and Techonology of State of Ceara - IFCE, ORCID:0000-0003-3710-1561

4 University of Tras-os-Montes and Alto Douro - UTAD, ORCID:0000-0001-6917-5093

*Corresponding author: re.passosm@gmail.com

Received: 7 July 2021, Accepted: 20 October 2021, Available online: 27 December 2021
\end{abstract}

\section{Introduction}

A recursive linear sequence has an infinite number of terms and is generated by a linear recurrence, called a recurrence formula, which allows you to calculate the terms of the sequence from its predecessors. Thus, in order to be able to calculate the terms of a sequence, it is necessary to know its initial terms. For mathematics, sequences are found in the area of number theory and have applicability in several areas.

In the mathematical scope, the Fibonacci sequence is the most explored sequence, however, the Padovan sequence is considered a prime Fibonacci sequence which is a linear and recurrent type sequence of third order, of integers. The Padovan Sequence, named after the Italian architect Richard Padovan (1935 - ?) [13], his work and contributions have important repercussions for research in Mathematics.

On the other hand, we have the Perrin sequence, which is a linear and recurrent sequence of integers and presents the same recurrence relation as the Padovan sequence, differing only in the terms of the sequence. This sequence was defined in 1899 by the French mathematician Olivier Raoul Perrin (1841-1910) [16]. Due to the similarity between the Padovan and Perrin sequences, in the works of $[2,6,12]$ properties and identities between these numbers are presented.

So we have the recurrences of the Padovan and Perrin sequences defined below.

Definition 1.1. The recurrence of the sequence of Padovan and Perrin, respectively, is given by:

$$
P_{n}=P_{n-2}+P_{n-3}, n \geqslant 3,
$$




$$
P e_{n}=P e_{n-2}+P e_{n-3}, n \geq 3
$$

being $P_{0}=P_{1}=P_{2}=1, P e_{0}=3, P e_{1}=0$ and $P e_{2}=2$ the initial conditions.

Since these sequences have the same recurrence relation, we can perform algebraic operations in order to obtain the characteristic polynomial of these sequences [1].

Definition 1.2. The characteristic polynomial of the Padovan and Perrin Sequence is defined as:

$$
x^{3}-x-1=0
$$

having three roots, two complex and one real.

And yet, in the literature of pure mathematics, there is the set of hybrid numbers, defined by [10], which presents the complex, hyperbolic and dual numbers together, combined with each other.

Definition 1.3. A hybrid number is defined as:

$$
\mathbb{K}=\left\{z=a+b i+c \varepsilon+d h: a, b, c, d \in \mathbb{R}, i^{2}=-1, \varepsilon^{2}=0, h^{2}=1, i h=-h i=\varepsilon+i\right\}
$$

From the definition of hybrid numbers, it is possible to perform some operations with these numbers, namely: addition, subtraction, multiplication by scalar. As for the multiplication between two hybrid numbers, this product is obtained by distributing the terms to the right, preserving the order of multiplication of the units and using the equalities $i^{2}=-1, \varepsilon^{2}=$ $0, h^{2}=1, i h=-h i=\varepsilon+i$.

From the multiplication of the imaginary units, we can present the table of the multiplication of a hybrid number, as shown in the Table 1.

\begin{tabular}{|c|c|c|c|c|}
\hline$\cdot$ & 1 & $i$ & $\varepsilon$ & $h$ \\
\hline 1 & 1 & $i$ & $\varepsilon$ & $h$ \\
\hline$i$ & $i$ & -1 & $1-h$ & $\varepsilon+i$ \\
\hline$\varepsilon$ & $\varepsilon$ & $1+h$ & 0 & $-\varepsilon$ \\
\hline$h$ & $h$ & $-\varepsilon-i$ & $\varepsilon$ & 1 \\
\hline
\end{tabular}

Table 1. Multiplication table for $\mathbb{K}$.

Furthermore, from the hybrid numbers it is possible to present their conjugate, denoted by $\bar{z}$ and is defined as

$$
\bar{z}=a-b i-c \varepsilon-d h
$$

and the real number

$$
C(z)=z \bar{z}=\bar{z} z=a^{2}+(b-c)^{2}-c^{2}-d^{2}=a^{2}+b^{2}-2 b c-d^{2}
$$

is called the hybrid number character, where the root of the absolute value of that real number will be the hybrid number norm. $z$, so we have to: $\|z\|=\sqrt{|C(z)|}$.

From the linear recursive sequences and the hybrid numbers, the hybridization process of the sequences is then carried out, as seen in $[4,7,8,9,14,15]$. For the Padovan and Perrin sequence we can define these numbers as:

Definition 1.4. The hybrid numbers of Padovan and Perrin, denoted by $P H_{n}$ and $P e H_{n}$, are defined as:

$$
\begin{gathered}
P H_{n}=P_{n}+P_{n+1} i+P_{n+2} \varepsilon+P_{n+3} h, \\
P e H_{n}=P e_{n}+P e_{n+1} i+P e_{n+2} \varepsilon+P e_{n+3} h,
\end{gathered}
$$

where $\mathrm{PH}_{0}=1+i+\varepsilon+2 h$ and $\mathrm{PH}_{1}=1+i+2 \varepsilon+2 h$ and $\mathrm{PH}_{2}=1+2 i+2 \varepsilon+3$ h the initial conditions for hybrids of Padovan and $\mathrm{PeH}_{0}=3+2 \varepsilon+3 h$ and $\mathrm{PeH}_{1}=2 i+3 \varepsilon+2 h$ and $\mathrm{PeH}_{2}=2+3 i+2 \varepsilon+5 h$ the initial conditions for Perrin hybrids.

Based on the work of [11], we can present the hybrid Padovan matrix form $\left(Q_{n}\right)$ given by:

$$
Q_{n}=\left[\begin{array}{ccc}
P H_{n+2} & P H_{n+1} & P H_{n} \\
P H_{n+3} & P H_{n+2} & P H_{n+1} \\
P H_{n+1} & P H_{n} & P H_{n-1}
\end{array}\right], \text { for } n \geqslant 1 \text {. }
$$


That satisfies equality $Q_{n}=U^{n} Q$, where:

$$
U=\left[\begin{array}{lll}
0 & 1 & 0 \\
1 & 0 & 1 \\
1 & 0 & 0
\end{array}\right], Q=\left[\begin{array}{ccc}
1+2 i+2 \varepsilon+3 h & 1+i+2 \varepsilon+2 h & 1+i+\varepsilon+2 h \\
2+2 i+3 \varepsilon+4 h & 1+2 i+2 \varepsilon+3 h & 1+i+2 \varepsilon+2 h \\
1+i+2 \varepsilon+2 h & 1+i+\varepsilon+2 h & i+\varepsilon+h
\end{array}\right]
$$

So, for $n=1$, you have that:

$$
\begin{aligned}
Q_{1}=U^{1} Q & =\left[\begin{array}{lll}
0 & 1 & 0 \\
1 & 0 & 1 \\
1 & 0 & 0
\end{array}\right]\left[\begin{array}{ccc}
1+2 i+2 \varepsilon+3 h & 1+i+2 \varepsilon+2 h & 1+i+\varepsilon+2 h \\
2+2 i+3 \varepsilon+4 h & 1+2 i+2 \varepsilon+3 h & 1+i+2 \varepsilon+2 h \\
1+i+2 \varepsilon+2 h & 1+i+\varepsilon+2 h & i+\varepsilon+h
\end{array}\right] \\
& =\left[\begin{array}{lll}
2+2 i+3 \varepsilon+4 h & 1+2 i+2 \varepsilon+3 h & 1+i+2 \varepsilon+2 h \\
2+3 i+4 \varepsilon+5 h & 2+2 i+3 \varepsilon+4 h & 1+2 i+2 \varepsilon+3 h \\
1+2 i+2 \varepsilon+3 h & 1+i+2 \varepsilon+2 h & 1+i+\varepsilon+2 h
\end{array}\right] \\
& =\left[\begin{array}{lll}
P H_{3} & P H_{2} & P H_{1} \\
P H_{4} & P H_{3} & P H_{2} \\
P H_{2} & P H_{1} & P H_{0}
\end{array}\right]
\end{aligned}
$$

Assuming it is valid for $n=k,(k \in \mathbb{Z})$ :

$$
\begin{aligned}
Q_{k}=U^{k} Q= & {\left[\begin{array}{lll}
0 & 1 & 0 \\
1 & 0 & 1 \\
1 & 0 & 0
\end{array}\right]^{k}\left[\begin{array}{ccc}
1+2 i+2 \varepsilon+3 h & 1+i+2 \varepsilon+2 h & 1+i+\varepsilon+2 h \\
2+2 i+3 \varepsilon+4 h & 1+2 i+2 \varepsilon+3 h & 1+i+2 \varepsilon+2 h \\
1+i+2 \varepsilon+2 h & 1+i+\varepsilon+2 h & i+\varepsilon+h
\end{array}\right] } \\
= & {\left[\begin{array}{lll}
P_{n-2} & P_{n-3} & P_{n-4} \\
P_{n-1} & P_{n-2} & P_{n-3} \\
P_{n-3} & P_{n-4} & P_{n-5}
\end{array}\right]\left[\begin{array}{ccc}
1+2 i+2 \varepsilon+3 h & 1+i+2 \varepsilon+2 h & 1+i+\varepsilon+2 h \\
2+2 i+3 \varepsilon+4 h & 1+2 i+2 \varepsilon+3 h & 1+i+2 \varepsilon+2 h \\
1+i+2 \varepsilon+2 h & 1+i+\varepsilon+2 h & i+\varepsilon+h
\end{array}\right] } \\
= & {\left[\begin{array}{ccc}
P H_{k+2} & P H_{k+1} & P H_{k} \\
P H_{k+3} & P H_{k+2} & P H_{k+1} \\
P H_{k+1} & P H_{k} & P H_{k-1}
\end{array}\right] }
\end{aligned}
$$

In this way, it is shown that it is valid for $n=k+1,(k \in \mathbb{Z})$ :

$$
\begin{aligned}
Q_{k+1}=U^{k+1} Q & =\left[\begin{array}{lll}
0 & 1 & 0 \\
1 & 0 & 1 \\
1 & 0 & 0
\end{array}\right]^{k+1}\left[\begin{array}{ccc}
1+2 i+2 \varepsilon+3 h & 1+i+2 \varepsilon+2 h & 1+i+\varepsilon+2 h \\
2+2 i+3 \varepsilon+4 h & 1+2 i+2 \varepsilon+3 h & 1+i+2 \varepsilon+2 h \\
1+i+2 \varepsilon+2 h & 1+i+\varepsilon+2 h & i+\varepsilon+h
\end{array}\right] \\
& =\left[\begin{array}{lll}
0 & 1 & 0 \\
1 & 0 & 1 \\
1 & 0 & 0
\end{array}\right]^{k}\left[\begin{array}{lll}
0 & 1 & 0 \\
1 & 0 & 1 \\
1 & 0 & 0
\end{array}\right]\left[\begin{array}{ccc}
1+2 i+2 \varepsilon+3 h & 1+i+2 \varepsilon+2 h & 1+i+\varepsilon+2 h \\
2+2 i+3 \varepsilon+4 h & 1+2 i+2 \varepsilon+3 h & 1+i+2 \varepsilon+2 h \\
1+i+2 \varepsilon+2 h & 1+i+\varepsilon+2 h & i+\varepsilon+h
\end{array}\right] \\
& =\left[\begin{array}{lll}
P_{n-2} & P_{n-3} & P_{n-4} \\
P_{n-1} & P_{n-2} & P_{n-3} \\
P_{n-3} & P_{n-4} & P_{n-5}
\end{array}\right]\left[\begin{array}{ccc}
0 & 1 & 0 \\
1 & 0 & 1 \\
1 & 0 & 0
\end{array}\right]\left[\begin{array}{ccc}
1+2 i+2 \varepsilon+3 h & 1+i+2 \varepsilon+2 h & 1+i+\varepsilon+2 h \\
2+2 i+3 \varepsilon+4 h & 1+2 i+2 \varepsilon+3 h & 1+i+2 \varepsilon+2 h \\
1+i+2 \varepsilon+2 h & 1+i+\varepsilon+2 h & i+\varepsilon+h
\end{array}\right] \\
& =\left[\begin{array}{llll}
P_{n-1} & P_{n-2} & P_{n-3} \\
P_{n} & P_{n-1} & P_{n-2} \\
P_{n-2} & P_{n-3} & P_{n-4}
\end{array}\right]\left[\begin{array}{ccc}
1+2 i+2 \varepsilon+3 h & 1+i+2 \varepsilon+2 h & 1+i+\varepsilon+2 h \\
2+2 i+3 \varepsilon+4 h & 1+2 i+2 \varepsilon+3 h & 1+i+2 \varepsilon+2 h \\
1+i+2 \varepsilon+2 h & 1+i+\varepsilon+2 h & i+\varepsilon+h
\end{array}\right] \\
& =\left[\begin{array}{llll}
P H_{k+3} & P H_{k+2} & P H_{k+1} \\
P H_{k+4} & P H_{k+3} & P H_{k+2} \\
P H_{k+2} & P H_{k+1} & P H_{k}
\end{array}\right]
\end{aligned}
$$

Furthermore, the Perrin hybrid matrix $\left(W_{n}\right)$ is given by:

$$
W_{n}=\left[\begin{array}{ccc}
\mathrm{PeH}_{n+2} & \mathrm{PeH}_{n+1} & \mathrm{PeH}_{n} \\
\mathrm{PeH}_{n+3} & \mathrm{PeH}_{n+2} & \mathrm{PeH}_{n+1} \\
\mathrm{PeH}_{n+1} & \mathrm{PeH}_{n} & \mathrm{PeH}_{n-1}
\end{array}\right] \text {, for } n \geqslant 1 .
$$


That satisfies equality $W_{n}=A^{n} W$, where

$$
A=\left[\begin{array}{ccc}
0 & 1 & 0 \\
1 & 0 & 1 \\
1 & 0 & 0
\end{array}\right], W=\left[\begin{array}{ccc}
2+3 i+2 \varepsilon+5 h & 2 i+3 \varepsilon+2 h & 3+2 \varepsilon+3 h \\
3+2 i+5 \varepsilon+5 h & 2+3 i+2 \varepsilon+5 h & 2 i+3 \varepsilon+2 h \\
2 i+3 \varepsilon+2 h & 3+2 \varepsilon+3 h & -1+3 i+2 h
\end{array}\right] .
$$

With this, based on what was previously presented and based on the work of [17], in this work, we will present several identities on Padovan and Perrin hybrid numbers, their extension to hybrids with negative indices and identities around them.

\section{Results}

Based on the definitions seen in the introduction referring to the hybrid numbers of Padovan and Perrin, some theorems are then developed with the aim of carrying out a new investigative study on these numbers.

Definition 2.1. Hybrid numbers of Padovan and Perrin with negative indices are defined by:

$$
\begin{aligned}
P H_{-n} & =P_{-n}+P_{-n+1} i+P_{-n+2} \varepsilon+P_{-n+3} h \\
P e H_{-n} & =P e_{-n}+P e_{-n+1} i+P e_{-n+2} \varepsilon+P e_{-n+3} h
\end{aligned}
$$

Based on this, we can obtain the binomial sums of the Padovan hybrid numbers in the following theorem.

Theorem 2.2. The following identities are valid:

(i) $\sum_{k=0}^{n}\left(\begin{array}{l}n \\ k\end{array}\right) P H_{k}=P_{3 n}$,

(ii) $\sum_{k=0}^{n}\left(\begin{array}{l}n \\ k\end{array}\right) P H_{k+1}=P H_{3 n+1}$,

Proof. (i) According to Binet's formula of Padovan hybrid numbers $P H_{n}=A x_{1}^{n}+B x_{2}^{n}+C x_{3}^{n}$, one has that:

$$
\begin{aligned}
& \sum_{k=0}^{n}\left(\begin{array}{l}
n \\
k
\end{array}\right) P H_{k}=\sum_{k=0}^{n}\left(\begin{array}{l}
n \\
k
\end{array}\right)\left(A x_{1}^{k}+B x_{2}^{k}+C x_{3}^{k}\right) \\
& A \sum_{k=0}^{n}\left(\begin{array}{l}
n \\
k
\end{array}\right) x_{1}^{k}+B \sum_{k=0}^{n}\left(\begin{array}{l}
n \\
k
\end{array}\right) x_{2}^{k}+C \sum_{k=0}^{n}\left(\begin{array}{l}
n \\
k
\end{array}\right) x_{3}^{k} \\
& A\left(1+x_{1}\right)^{n}+B\left(1+x_{2}\right)^{n}+C\left(1+x_{3}\right)^{n} .
\end{aligned}
$$

Considering the infinite interactions of the cubic roots given by the expression $\psi=\sqrt[3]{1+\sqrt[3]{1+} \sqrt[3]{1+} \ldots}$, one can establish the relation $\psi^{3}=1+\psi$, where $\psi$ represents the real Padovan root [5]. Hence, $1+x_{1}=x_{1}^{3}, 1+x_{2}=x_{2}^{3}$ and $1+x_{3}=x_{3}^{3}$. With that, $\sum_{k=0}^{n}\left(\begin{array}{l}n \\ k\end{array}\right) P H_{k}=P H_{3 n}$.

(ii) Similarly to demonstration (i), this Identity can be validated.

Thus, we have the binomial sums of the Perrin hybrid numbers in the following proposition. Since the proof of these sums is similar to the Padovan hybrid numbers discussed in the previous Theorem, we omit the proof.

Theorem 2.3. The following identities are valid:

(i) $\sum_{k=0}^{n}\left(\begin{array}{l}n \\ k\end{array}\right) \mathrm{PeH}_{k}=\mathrm{PeH}_{3 n}$,

(ii) $\sum_{k=0}^{n}\left(\begin{array}{l}n \\ k\end{array}\right) \mathrm{PeH}_{k+1}=\mathrm{PeH}_{3 n+1}$.

Theorem 2.4. For $m \geqslant 3, n \geqslant 3$, one can:

$\mathrm{PH}_{n+2} \mathrm{PH}_{m}+\mathrm{PH}_{n+1} \mathrm{PH}_{m+1}+\mathrm{PH}_{n} \mathrm{PH}_{m-1}=\mathrm{PH}_{n+m}+\mathrm{PH}_{n+m+1} i+\mathrm{PH}_{n+m+2} \varepsilon+P H_{n+m+3} h$.

Proof. According to the matrix form of the Padovan hybrid numbers:

$$
\begin{array}{r}
U=\left[\begin{array}{lll}
0 & 1 & 0 \\
1 & 0 & 1 \\
1 & 0 & 0
\end{array}\right], Q=\left[\begin{array}{ccc}
1+2 i+2 \varepsilon+3 h & 1+i+2 \varepsilon+2 h & 1+i+\varepsilon+2 h \\
2+2 i+3 \varepsilon+4 h & 1+2 i+2 \varepsilon+3 h & 1+i+2 \varepsilon+2 h \\
1+i+2 \varepsilon+2 h & 1+i+\varepsilon+2 h & i+\varepsilon+h
\end{array}\right] \\
U^{n}=\left[\begin{array}{ccc}
P_{n-2} & P_{n-3} & P_{n-4} \\
P_{n-1} & P_{n-2} & P_{n-3} \\
P_{n-3} & P_{n-4} & P_{n-5}
\end{array}\right]
\end{array}
$$


Resulting in $Q_{n}=U^{n} Q$ :

$$
\left[\begin{array}{ccc}
P H_{n+2} & P H_{n+1} & P H_{n} \\
P H_{n+3} & P H_{n+2} & P H_{n+1} \\
P H_{n+1} & P H_{n} & P H_{n-1}
\end{array}\right]
$$

Thus, performing $\left(Q_{n}\right)\left(Q_{m}\right)$, one can equal the term $a_{13}$, obtaining:

$$
\left[\begin{array}{ccc}
P H_{n+2} & P H_{n+1} & P H_{n} \\
P H_{n+3} & P H_{n+2} & P H_{n+1} \\
P H_{n+1} & P H_{n} & P H_{n-1}
\end{array}\right]\left[\begin{array}{ccc}
P H_{m+2} & P H_{m+1} & P H_{m} \\
P H_{m+3} & P H_{m+2} & P H_{m+1} \\
P H_{m+1} & P H_{m} & P H_{m-1}
\end{array}\right]=P H_{n+2} P H_{m}+P H_{n+1} P H_{m+1}+P H_{n} P H_{m-1} .
$$

By definition, one has to $\mathrm{PH}_{n}=\mathrm{PH}_{n+m}+\mathrm{PH}_{n+m+1} i+P \mathrm{H}_{n+m+2} \varepsilon+P H_{n+m+3} h$.

Soon:

$$
P H_{n+2} P H_{m}+P H_{n+1} P H_{m+1}+P H_{n} P H_{m-1}=P H_{n+m}+P H_{n+m+1} i+P H_{n+m+2} \varepsilon+P H_{n+m+3} h .
$$

Theorem 2.5. For $m \geqslant 0, n \geqslant 0$, one has to:

$\mathrm{PeH}_{n+2} \mathrm{PeH}_{m}+\mathrm{PeH}_{n+1} \mathrm{PeH}_{m+1}+\mathrm{PeH}_{n} \mathrm{PeH}_{m-1}=\mathrm{PeH}_{n+m}+\mathrm{PeH}_{n+m+1} i+\mathrm{PeH}_{n+m+2} \varepsilon+\mathrm{PeH}_{n+m+3} h$.

Proof. According to the matrix form of Perrin's hybrid numbers:

$$
\begin{array}{r}
A=\left[\begin{array}{lll}
0 & 1 & 0 \\
1 & 0 & 1 \\
1 & 0 & 0
\end{array}\right], W=\left[\begin{array}{ccc}
2+3 i+2 \varepsilon+5 h & 2 i+3 \varepsilon+2 h & 3+2 \varepsilon+3 h \\
3+2 i+5 \varepsilon+5 h & 2+3 i+2 \varepsilon+5 h & 2 i+3 \varepsilon+2 h \\
2 i+3 \varepsilon+2 h & 3+2 \varepsilon+3 h & -1+3 i+2 h
\end{array}\right] \\
A^{n} W=\left[\begin{array}{ccc}
\mathrm{PeH}_{n+2} & \mathrm{PeH}_{n+1} & \mathrm{PeH} \\
\mathrm{PeH}_{n+3} & \mathrm{PeH}_{n+2} & \mathrm{PeH}_{n+1} \\
\mathrm{PeH}_{n+1} & \mathrm{PeH}_{n} & \mathrm{PeH}_{n-1}
\end{array}\right]
\end{array}
$$

Thus, performing $\left(W_{n}\right)\left(W_{m}\right)$, one can equal the term $a_{13}$, obtaining:

$$
\left[\begin{array}{ccc}
\mathrm{PeH}_{n+2} & \mathrm{PeH}_{n+1} & \mathrm{PeH}_{n} \\
\mathrm{PeH}_{n+3} & \mathrm{PeH}_{n+2} & \mathrm{PeH}_{n+1} \\
\mathrm{PeH}_{n+1} & \mathrm{PeH}_{n} & \mathrm{PeH}_{n-1}
\end{array}\right]\left[\begin{array}{ccc}
\mathrm{PeH}_{m+2} & \mathrm{PeH}_{m+1} & \mathrm{PeH}_{m} \\
\mathrm{PeH}_{m+3} & \mathrm{PeH}_{m+2} & \mathrm{PeH}_{m+1} \\
\mathrm{PeH}_{m+1} & \mathrm{PeH}_{m} & \mathrm{PeH}_{m-1}
\end{array}\right]=\mathrm{PeH}_{n+2} \mathrm{PeH}_{m}+\mathrm{PeH}_{n+1} \mathrm{PeH}_{m+1}+\mathrm{PeH}_{n} \mathrm{PeH}_{m-1}
$$

By definition, one has to $\mathrm{PeH}_{n}=\mathrm{PeH}_{n+m}+\mathrm{PeH}_{n+m+1} i+\mathrm{PeH}_{n+m+2} \varepsilon+\mathrm{PeH}_{n+m+3} h$. Soon:

$$
\mathrm{PeH}_{n+2} \mathrm{PeH}_{m}+\mathrm{PeH}_{n+1} \mathrm{PeH}_{m+1}+\mathrm{PeH}_{n} \mathrm{PeH}_{m-1}=\mathrm{PeH}_{n+m}+\mathrm{PeH}_{n+m+1} i+\mathrm{PeH}_{n+m+2} \varepsilon+\mathrm{PeH}_{n+m+3} h .
$$

Theorem 2.6. For $m \geqslant 3, n \geqslant 3$, one has to:

(i) $P H_{m+n}-P H_{m+n+1} i-P H_{m+n+2} \varepsilon-P H_{m+n+3} h=\overline{P H}_{m+2} P H_{n}+\overline{P H}_{m+1} P H_{n+1}+\overline{P H}_{m} P H_{n-1}$, (ii) $\overline{P H}_{n+m}+\overline{P H}_{n+m+1} i+\overline{P H}_{n+m+2} \varepsilon+\overline{P H}_{n+m+3} h=P H_{m+2} \overline{P H}_{n}+P H_{m+1} \overline{P H}_{n+1}+P H_{m} \overline{P H}_{n-1}$.

Proof. Using the conjugate of the matrix $Q$, called $\bar{Q}$, where:

$Q=\left[\begin{array}{ccc}1+2 i+2 \varepsilon+3 h & 1+i+2 \varepsilon+2 h & 1+i+\varepsilon+2 h \\ 2+2 i+3 \varepsilon+4 h & 1+2 i+2 \varepsilon+3 h & 1+i+2 \varepsilon+2 h \\ 1+i+2 \varepsilon+2 h & 1+i+\varepsilon+2 h & i+\varepsilon+h\end{array}\right]$.

Thus, performing:

$$
\begin{aligned}
U^{n} \bar{Q} & =\left[\begin{array}{lll}
P_{n-2} & P_{n-3} & P_{n-4} \\
P_{n-1} & P_{n-2} & P_{n-3} \\
P_{n-3} & P_{n-4} & P_{n-5}
\end{array}\right]\left[\begin{array}{ccc}
1-2 i-2 \varepsilon-3 h & 1-i-2 \varepsilon-2 h & 1-i-\varepsilon-2 h \\
2-2 i-3 \varepsilon-4 h & 1-2 i-2 \varepsilon-3 h & 1-i-2 \varepsilon-2 h \\
1-i-2 \varepsilon-2 h & 1-i-\varepsilon-2 h & i-\varepsilon-h
\end{array}\right] \\
& =\left[\begin{array}{lll}
\overline{P H}_{n+2} & \overline{P H}_{n+1} & \overline{P H}_{n} \\
\overline{P H}_{n+3} & \overline{P H}_{n+2} & \overline{P H}_{n+1} \\
\overline{P H}_{n+1} & \overline{P H}_{n} & \overline{P H}_{n-1}
\end{array}\right] .
\end{aligned}
$$


Thus, considering the element $a_{13}$, it can be said that:

(i) $\bar{Q}\left(U^{m+n} Q\right)=\left(\bar{Q} U^{m}\right)\left(U^{n} Q\right)$,

$$
\begin{aligned}
& \bar{Q}\left(U^{m+n} Q\right)=\bar{Q}\left[\begin{array}{lll}
P_{n+m-2} & P_{n+m-3} & P_{n+m-4} \\
P_{n+m-1} & P_{n+m-2} & P_{n+m-3} \\
P_{n+m-3} & P_{n+m-4} & P_{n+m-5}
\end{array}\right]\left[\begin{array}{ccc}
1+2 i+2 \varepsilon+3 h & 1+i+2 \varepsilon+2 h & 1+i+\varepsilon+2 h \\
2+2 i+3 \varepsilon+4 h & 1+2 i+2 \varepsilon+3 h & 1+i+2 \varepsilon+2 h \\
1+i+2 \varepsilon+2 h & 1+i+\varepsilon+2 h & i+\varepsilon+h
\end{array}\right] \\
& =\left[\begin{array}{ccc}
1-2 i-2 \varepsilon-3 h & 1-i-2 \varepsilon-2 h & 1-i-\varepsilon-2 h \\
2-2 i-3 \varepsilon-4 h & 1-2 i-2 \varepsilon-3 h & 1-i-2 \varepsilon-2 h \\
1-i-2 \varepsilon-2 h & 1-i-\varepsilon-2 h & i-\varepsilon-h
\end{array}\right]\left[\begin{array}{ccc}
P H_{n+m+2} & P H_{n+m+1} & P H_{n+m} \\
P H_{n+m+3} & P H_{n+m+2} & P H_{n+m+1} \\
P H_{n+m+1} & P H_{n+m} & P H_{n+m-1}
\end{array}\right] \\
& =\left[\begin{array}{ccc}
\overline{P H}_{n+m+2} & \overline{P H}_{n+m+1} & \overline{P H}_{n+m} \\
\overline{P H}_{n+m+3} & \overline{P H}_{n+m+2} & \overline{P H}_{n+m+1} \\
\overline{P H}_{n+m+1} & \overline{P H}_{n+m} & \overline{P H}_{n+m-1}
\end{array}\right] \text {. } \\
& \left(\bar{Q} U^{m}\right)\left(U^{n} Q\right)=\left[\begin{array}{ccc}
1-2 i-2 \varepsilon-3 h & 1-i-2 \varepsilon-2 h & 1-i-\varepsilon-2 h \\
2-2 i-3 \varepsilon-4 h & 1-2 i-2 \varepsilon-3 h & 1-i-2 \varepsilon-2 h \\
1-i-2 \varepsilon-2 h & 1-i-\varepsilon-2 h & i-\varepsilon-h
\end{array}\right]\left[\begin{array}{ccc}
P_{m-2} & P_{m-3} & P_{m-4} \\
P_{m-1} & P_{m-2} & P_{m-3} \\
P_{m-3} & P_{m-4} & P_{m-5}
\end{array}\right]\left(U^{n} Q\right) \\
& =\left[\begin{array}{ccc}
\overline{P H}_{m+2} & \overline{P H}_{m+1} & \overline{P H}_{m} \\
\overline{P H}_{m+3} & \overline{P H}_{m+2} & \overline{P H}_{m+1} \\
\overline{P H}_{m+1} & \overline{P H}_{m} & \overline{P H}_{m-1}
\end{array}\right]\left[\begin{array}{ccc}
P_{n-2} & P_{n-3} & P_{n-4} \\
P_{n-1} & P_{n-2} & P_{n-3} \\
P_{n-3} & P_{n-4} & P_{n-5}
\end{array}\right]\left[\begin{array}{ccc}
1+2 i+2 \varepsilon+3 h & 1+i+2 \varepsilon+2 h & 1+i+\varepsilon+2 h \\
2+2 i+3 \varepsilon+4 h & 1+2 i+2 \varepsilon+3 h & 1+i+2 \varepsilon+2 h \\
1+i+2 \varepsilon+2 h & 1+i+\varepsilon+2 h & i+\varepsilon+h
\end{array}\right] \\
& =\left[\begin{array}{ccc}
\overline{P H}_{m+2} & \overline{P H}_{m+1} & \overline{P H}_{m} \\
\overline{P H}_{m+3} & \overline{P H}_{m+2} & \overline{P H}_{m+1} \\
\overline{P H}_{m+1} & \overline{P H}_{m} & \overline{P H}_{m-1}
\end{array}\right]\left[\begin{array}{ccc}
P H_{n+2} & P H_{n+1} & P H_{n} \\
P H_{n+3} & P H_{n+2} & P H_{n+1} \\
P H_{n+1} & P H_{n} & P H_{n-1}
\end{array}\right]
\end{aligned}
$$

(ii) $\left(Q U^{m+n}\right) \bar{Q}=\left(Q U^{m}\right)\left(U^{n} \bar{Q}\right)$,

$$
\begin{aligned}
& \left(Q U^{m+n}\right) \bar{Q}=\left[\begin{array}{ccc}
1+2 i+2 \varepsilon+3 h & 1+i+2 \varepsilon+2 h & 1+i+\varepsilon+2 h \\
2+2 i+3 \varepsilon+4 h & 1+2 i+2 \varepsilon+3 h & 1+i+2 \varepsilon+2 h \\
1+i+2 \varepsilon+2 h & 1+i+\varepsilon+2 h & i+\varepsilon+h
\end{array}\right]\left[\begin{array}{ccc}
P_{m+n-2} & P_{m+n-3} & P_{m+n-4} \\
P_{m+n-1} & P_{m+n-2} & P_{m+n-3} \\
P_{m+n-3} & P_{m+n-4} & P_{m+n-5}
\end{array}\right] \bar{Q} \\
& =\left[\begin{array}{ccc}
P H_{m+n+2} & P H_{m+n+1} & P H_{m+n} \\
P H_{m+n+3} & P H_{m+n+2} & P H_{m+n+1} \\
P H_{m+n+1} & P H_{m+n} & P H_{m+n-1}
\end{array}\right]\left[\begin{array}{ccc}
1-2 i-2 \varepsilon-3 h & 1-i-2 \varepsilon-2 h & 1-i-\varepsilon-2 h \\
2-2 i-3 \varepsilon-4 h & 1-2 i-2 \varepsilon-3 h & 1-i-2 \varepsilon-2 h \\
1-i-2 \varepsilon-2 h & 1-i-\varepsilon-2 h & i-\varepsilon-h
\end{array}\right] \\
& =\left[\begin{array}{ccc}
\overline{P H}_{m+n+2} & \overline{P H}_{m+n+1} & \overline{P H}_{m+n} \\
\overline{P H}_{m+n+3} & \overline{P H}_{m+n+2} & \overline{P H}_{m+n+1} \\
\overline{P H}_{m+n+1} & \overline{P H}_{m+n} & \overline{P H}_{m+n-1}
\end{array}\right]
\end{aligned}
$$

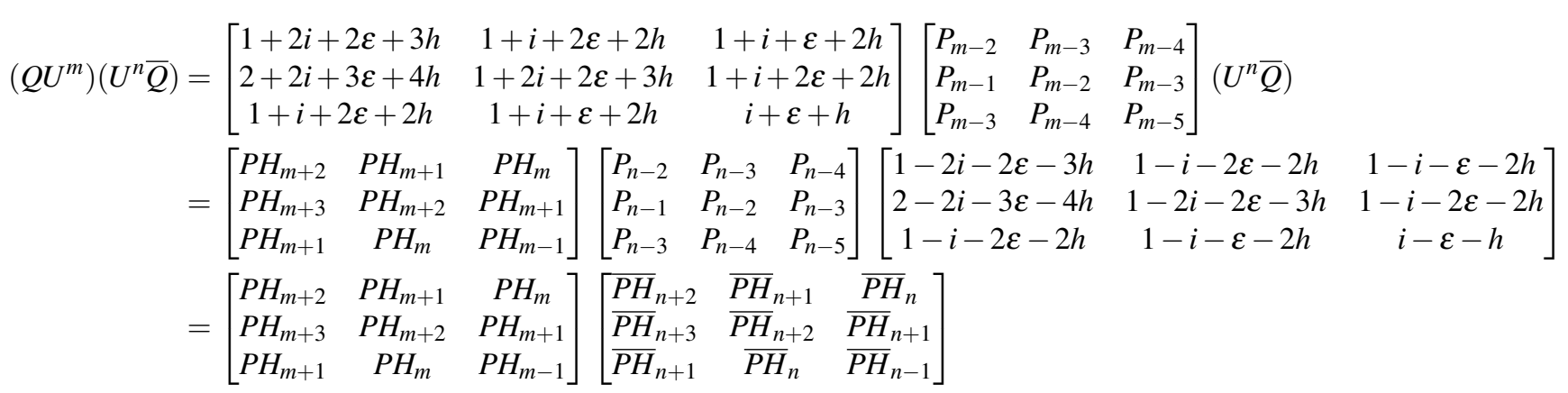

Theorem 2.7. For $m \geqslant 0, n \geqslant 0$, one has to:

(i) $\mathrm{PeH}_{m+n}-\mathrm{PeH}_{m+n+1} i-\mathrm{PeH}_{m+n+2} \varepsilon-\mathrm{PeH}_{m+n+3} h=\overline{\mathrm{PeH}}_{m+2} \mathrm{PeH}_{n}+\overline{\mathrm{PeH}}_{m+1} \mathrm{PeH} \mathrm{H}_{n+1}+\overline{\mathrm{PeH}}_{m} \mathrm{PeH} \mathrm{H}_{n-1}$, (ii) $\overline{\mathrm{PeH}}_{n+m}+\overline{\mathrm{PeH}}_{n+m+1} i+\overline{\mathrm{PeH}}_{n+m+2} \varepsilon+\overline{\mathrm{PeH}}_{n+m+3} h=\mathrm{PeH}_{m+2} \overline{\mathrm{PeH}}_{n}+\mathrm{PeH}_{m+1} \overline{\mathrm{PeH}}_{n+1}+\mathrm{PeH} \overline{\mathrm{PeH}}_{n-1}$. 
Proof. Using the conjugate of the matrix $W$, called $\bar{W}$, where:

$W=\left[\begin{array}{ccc}2+3 i+2 \varepsilon+5 h & 2 i+3 \varepsilon+2 h & 3+2 \varepsilon+3 h \\ 3+2 i+5 \varepsilon+5 h & 2+3 i+2 \varepsilon+5 h & 2 i+3 \varepsilon+2 h \\ 2 i+3 \varepsilon+2 h & 3+2 \varepsilon+3 h & -1+3 i+2 h\end{array}\right]$.

Thus, performing:

$$
A^{n} \bar{W}=\left[\begin{array}{lll}
0 & 1 & 0 \\
1 & 0 & 1 \\
1 & 0 & 0
\end{array}\right]^{n}\left[\begin{array}{ccc}
2-3 i-2 \varepsilon-5 h & 2 i-3 \varepsilon-2 h & 3-2 \varepsilon-3 h \\
3-2 i-5 \varepsilon-5 h & 2-3 i-2 \varepsilon-5 h & 2 i-3 \varepsilon-2 h \\
2 i-3 \varepsilon-2 h & 3-2 \varepsilon-3 h & -1-3 i-2 h
\end{array}\right]=\left[\begin{array}{ccc}
\overline{P e H}_{n+2} & \overline{P e H}_{n+1} & \overline{P e H}_{n} \\
\overline{P e H}_{n+3} & \overline{P e H}_{n+2} & \overline{P e H}_{n+1} \\
\overline{P e H}_{n+1} & \overline{P e H}_{n} & \overline{P e H}_{n-1}
\end{array}\right] .
$$

Thus, considering the element $a_{13}$, it can be said that:

(i) $\bar{W}\left(A^{m+n} W\right)=\left(\bar{W} A^{m}\right)\left(A^{n} W\right)$,

(ii) $W\left(A^{m+n} \bar{W}\right)=\left(W A^{m}\right)\left(A^{n} \bar{W}\right)$.

\section{Conclusion}

This work carried out an investigative study around the hybrid numbers of Padovan and Perrin, developing new and proven theorems based on fundamental algebraic operations and their respective matrix forms. Thus, the results presented here have the bias of motivating further studies on the numbers of Padovan and Perrin hybrids, improving the investigation for other numerical sequences.

\section{Acknowledgements}

The development part of the research in Brazil had the financial support of the National Council for Scientific and Technological Development (CNPq) and the Coordination for the Improvement of Higher Education Personnel (CAPES).

The part of research development in Portugal is financed by National Funds through the Foundation for Science and Technology. IP (FCT), under the project UID/CED/00194/2020.

\section{Funding}

There is no funding for this work.

\section{Availability of data and materials}

Not applicable.

\section{Competing interests}

The authors declare that they have no competing interests.

\section{Author's contributions}

All authors contributed equally to the writing of this paper. All authors read and approved the final manuscript.

\section{References}

[1] J. Aarts, R. Fokkink, G. Kruijtzer, Morphic numbers, Nieuw Archief voor Wiskunde, 5(2) (2001), 56-58.

[2] K. Adegoke, Summation identities involving Padovan and Perrin numbers, arXiv preprint arXiv:1812.03241, (2018).

[3] P. Catarino, On k-pell hybrid numbers, J. Disc. Math. Sci. Cryp., Taylor \& Francis, (2019), 1-7.

[4] G. Cerda-Morales, Investigation of generalized hybrid Fibonacci numbers and their properties, arXiv preprint arXiv:1806.02231, (2018).

[5] R. Ferreira, Números mórficos, Dissertação de Mestrado Profissional em Matemática, Universidade Federal da Paraíba, João Pessoa, 2015.

[6] K. Khompungson, B. Rodjanadid, S. Sompong, Some matrices in term of Perrin and Padovan sequences, Thai J. Math., 17(3) (2019), 767-774. 
[7] M. C. dos S. Mangueira, et al., A generalização da forma matricial da sequência de Perrin, Revista Sergipana de Matemática e Educação Matemática, 5(1) (2020), 384-392.

[8] M. C. dos S. Mangueira, R. P. M. Vieira, F. R. V. Alves, P. M. M. C. Catarino, The hybrid numbers of Padovan and some identities, Annales Mathematicae Silesianaei 1(ahead-of-print), Sciendo, (2020).

[9] M. C. dos S. Mangueira, F. R. V. Alves, P. M. M. C. Catarino. Números híbridos de Mersenne, C.Q.D.-Revista Eletrônica Paulista de Matemática, 18 (2020), 1-11.

[10] M. Özdemir. Introducion to hybrid numbers, Adv. App. Cliff. Alg., 28 (2018).

[11] T. D. Senturk, et al. A Study on Horadam hybrid numbers, Turk. J. Math., 44(4) (2020), 1212-1221.

[12] K. Sokhuma, Matrices formula for Padovan and Perrin sequences, App. Math. Sci., 7(142) (2013), 7093-7096.

[13] I. Stewart, Tales of a neglected number, Scientific American, 274(6) (1996), 102-103, 1996.

[14] A. Szynal-Liana, The Horadam hybrid numbers, Discussiones Mathematicae-General Algebra and App., Sciendo, 38(1) (2018), 91-98.

[15] A. Szynal-Liana, I. Wloch. On Jacobsthal and Jacobsthal-Lucas hybrid numbers, In: Annales Mathematicae Silesianae, Sciendo, (2019), 276-283.

[16] R. Vieira, M. Mangueira, F. Alves, P. Catarino. Perrin n-dimensional relations, Fund. J. Math. App. 4(2) (2021), 100-109.

[17] N. Yilmaz. More identities on Fibonacci and Lucas hybrid numbersi Notes on Number Theory and Discrete Math. 27(2) (2021), 159-167. 